\title{
RACIONALIDADE E IRRACIONALIDADE NO PROCESSO DECISÓRIO DE CONSUMO $^{1}$
}

\section{RACIONALITY AND IRRACIONALITY IN THE CONSUMPTION DECISION MAKING PROCESS}

\author{
Claudio Felisoni de Angelo ${ }^{2}$ \\ Marcos Roberto Luppe ${ }^{3}$ \\ Nuno Manoel Martins Dias Fouto ${ }^{4}$
}

\begin{abstract}
RESUMO: Este trabalho tem por objetivo analisar o processo decisório de consumo considerando a evolução recente do mercado varejista no Brasil. Existem duas perspectivas. Para alguns importantes autores, os consumidores são racionais em suas escolhas. A idéia da racionalidade é a de que as pessoas consideram toda a informação que está disponível. Isto não significa que elas não cometem erros, mas significa que elas aprendem e ajustam seu comportamento durante a experiência. Se isto realmente é verdadeiro, isso implica que o passado explica completamente o nível atual de consumo. O segundo enfoque considera que os indivíduos não são capazes de escolher a melhor alternativa. Seguindo estas idéias, diferentes autores enfatizam que existem muitas limitações para as pessoas terem acesso a todas as informações disponíveis para alcançar decisões lógicas. Levando em consideração estes elementos teóricos e trabalhando com dados do mercado de consumo no Brasil, este trabalho conclue que as evidências conduzem para a hipótese da racionalidade limitada.
\end{abstract}

PALAVRAS-CHAVE: consumo, racionalidade, racionalidade limitada, expectativas adaptadas, expectativas racionais.

ABSTRACT: The purpose of this paper is to analyze rationality of consumption process considering the recent evolution of retail market in Brazil. There are two perspectives. For some important authors, consumers are rational in their choices. The idea of rationality is that people consider all information that is available. This does not mean that they do not committee mistakes, but it means that they learn and adjust their behavior throught out the experience. If this is really true it implies that the past explain completely the current consumption level. The second approach considers that individuals are not able to choose the best alternative. Following these ideas, many different authors stress that there are many limitations for people to access all information available to reach a logical decisions. Taking in consideration these theoretical elements and working with data from Brazil consumer market, this paper concludes that the evidences back the bounded racionality hypothesis.

KEYWORDS: consumption, racionality, bounded racionality, adaptative expectatives, rational expectatives.

\footnotetext{
${ }^{1}$ Artigo Recebido em 31.03.2009. Revisado por pares em 12.04.2010. Recomendado em 15.04.2010 por Leomar dos Santos Editor. Publicado em 08.09.2010.

Organização Responsável pelo periódico: Universidade regional de Blumenau - FURB - www.furb.br/rn

${ }^{2}$ Faculdade Economia, Administração e Contabilidade da Universidade de São Paulo - FEA/USP - cfa@usp.br

${ }^{3}$ Faculdade Economia, Administração e Contabilidade da Universidade de São Paulo - FEA/USP mluppe@usp.br

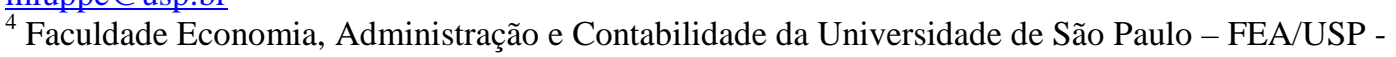
nunom@fia.com.br
} 


\section{INTRODUÇÃO}

A partir de 1994, com a edição do Plano Real, a economia brasileira entrou em uma nova etapa. A redução dos patamares inflacionários estabeleceu importantes condições para a expansão do mercado de consumo. Há concenso de que logo após a edição do plano, a queda dos preços permitiu uma expansão sensível do consumo. Posteriormente, entretanto, tal expansão foi obstada por uma forte recessão interna e um quadro internacional desfavorável. Essa situação só seria revertida anos mais tarde, ou seja, a partir de meados de 2004 (GIAMBIASI, 2005; IPEA, 2008).

O presente trabalho tem o propósito de estudar a evolução do consumo, mais especifícamente das vendas no varejo, neste ambiente da economia brasileira. Embora se pretendesse cobrir a análise a partir da vigência imediata do referido plano de estabilização monetária, por razões de disponibilidade de dados, reduziu-se o horizonte analítico cobrindo os meses de junho do ano 2000 a outubro de 2007.

Com base nessa amostra pretende-se revisitar o assunto da racionalidade do processo decisório em consumo, avaliando até que ponto as flutuações dessa variável podem ser associadas a comportamentos planejados devidamente antecipados pelos consumidores. Alternativamente a esse entendimento coloca-se a idéia de que os movimentos do consumo não são totalmente explicados pela experiência vivenciada nos períodos anteriores. Por essa perspectiva, dependendo das circunstâncias mais ou menos favoráveis se revelaria com maior ou menor intensidade a disposição dos indivíduos para o consumo. Essa visão se aproximaria da idéia que Schumpeter representou a disposição empreendedora, associando-a ao que ele denominou espírito animal, uma característica, segundo o autor, inerente à própria natureza humana (SCHUMPETER, 1934).

Há um grande número de estudos sobre essa questão, como por exemplo, Baumol (1968), Brockhaus (1982) e Bygrave (1989a; 1989b; 1993). Analisar, porém o assunto tomando como base a evolução recente da economia brasileira parece, entretanto, ser singular. A expansão do consumo pode ser melhor aclarada procurando determinar a previsibilidade dos agentes econômicos neste cenário e os efeitos das condições correntes no estímulo ou desestímulo à expansão ou contração das vendas varejistas. Há, portanto, implicações específicas associadas aos aspectos teóricos, confirmando ou não a natureza das decisões e efeitos sobre a gestão das organizações, que podem contribuir para a maior previsibilidade dos fluxos de consumo.

Embora as expectativas sejam um assunto de há muito presente nas preocupações da teoria econômica, a notoriedade do assunto somente se materializou a partir do trabalho de Milton Friedman (1957), sobre o processo de formação e acomodação das expectativas, o qual foi por ele denominado, expectativas adaptadas. Na seqüência, grande parte por inspiração das idéias seminais de Friedman, surgiu a hipótese das expectativas racionais (FRIEDMAN, 1957).

Tanto uma como outra, isto é, tanto as expectativas adaptadas como as expectativas racionais fundamentam-se no pressuposto clássico da racionalidade do consumidor.

Alternativamente a idéia da racionalidade como elemento básico propulsor do comportamento de consumo alinham-se os estudos de Amos Tversky e Daniel Kahneman, dois psicólogos com formação matemática. Por meio do desenho e aplicação de diversas heurísticas esses dois autores, bem como outros, têm procurado não apenas revelar a falta de racionalidade no processo decisório, mas também identificar as causas desse tipo de fenômeno (TVERSKY, KAHNEMAN, 1971, 1973, 1974; KAHNEMAN, TVERSKY, 1972, 1979). 


\section{RACIONALIDADE E IRRACIONALIDADE NO PROCESSO DECISÓRIO DE CONSUMO}

A contraposição destas concepções, amparada pelos dados da economia brasileira, segue-se nos próximos tópicos deste artigo organizado da seguinte forma. Na próxima seção é feito um breve relato da evolução da economia brasileira no horizonte de estudo, destacando aspectos relevantes ao entendimento da dinâmica do mercado de consumo. Em seguida, procura-se fazer uma revisão da literatura de modo a suscitar a modelagem mais adequada à análise da questão de pesquisa. Na seqüência, são apresentados os modelos analíticos e os correspondentes resultados. Finalmente na última seção, são sintetizadas as principais conclusões, apontadas as limitações e também sugeridas algumas possíveis extensões.

\section{A ECONOMIA BRASILEIRA E A EVOLUÇÃO DO CONSUMO}

O plano Real trouxe importantes mudanças para a economia brasileira. De modo especial em termos do mercado de consumo, duas alterações sobressaem para explicar as transformações que reconfiguram o ambiente para o consumo doméstico.

A queda da inflação trouxe consigo dois efeitos. Um diz respeito à redução do imposto inflacionário e de seu efeito negativo sobre a distribuição de renda dos indivíduos. O segundo, não menos significativo associa-se à mudança dos padrões de comparação de valor na compra de bens e na contratação de serviços.

A inflação é um imposto regressivo, pois retira renda dos indivíduos e a transfere para o governo. Tal transferência se dá de modo a penalizar exatamente as classes sociais menos abastadas. Desse modo, a manutenção de processos inflacionários fortes e duradouros contribui para a deterioração da distribuição de renda. Por essa via, a piora da distribuição de renda tende a inibir o ritmo de expansão do consumo interno.

Imaginando a sociedade dividida em apenas dois grupos, baixa e alta renda, sabe-se que o primeiro tem uma propensão marginal a consumir mais elevada que o segundo. Desse modo, um aumento da renda real por meio da redução da perda do poder aquisitivo tente a estimular o consumo.

A redução da inflação, alicerçada também na sobrevalorização cambial, aumentou as possibilidades de acesso das pessoas ao mercado de bens e serviços. Isto é, a operacionalização do Real permitiu no curto prazo uma sensível mudança no nível do consumo interno.

Porém a manutenção inflexível dos princípios que embasaram a edição do Real, particularmente no que diz respeito à âncora cambial, associada a crises financeiras no cenário internacional ocasionaram, nos últimos anos do governo FHC, uma forte recessão econômica com implicações sobre o emprego, a massa de salários e o consumo (GIAMBIASI, 2005).

Nesse ambiente assume o novo governo em 2003 com uma política econômica praticamente idêntica a anterior. A manutenção de nível de crescimento apenas ligeiramente mais elevado que o registrado nos últimos anos do governo anterior, e seguindo uma política fiscal e monetária bastante restritiva, o governo Lula consegue vencer as desconfianças suscitadas por sua tradição política.

Essa nova etapa, entretanto, apóia-se em uma substancial melhora das condições internacionais. As taxas de juros começam a declinar desde meados de 2003, quando se observa sinais mais nítidos de crescimento do consumo interno (IPEA, 2008).

A Figura 1 descreve o comportamento das vendas, renda e taxa de juros no período de junho de 2000 a outubro de 2007. Como se vê, as taxas de juros aumentaram até julho de 2003 e começaram a declinar de maneira consistente até o final do período de análise. A renda real, entretanto, caiu até mais ou menos dezembro de 2002 e a partir daí manteve-se praticamente inalterada. A série de vendas, por sua vez, mostra uma forte sazonalidade e uma tendência de expansão iniciada no segundo semestre de 2003. 


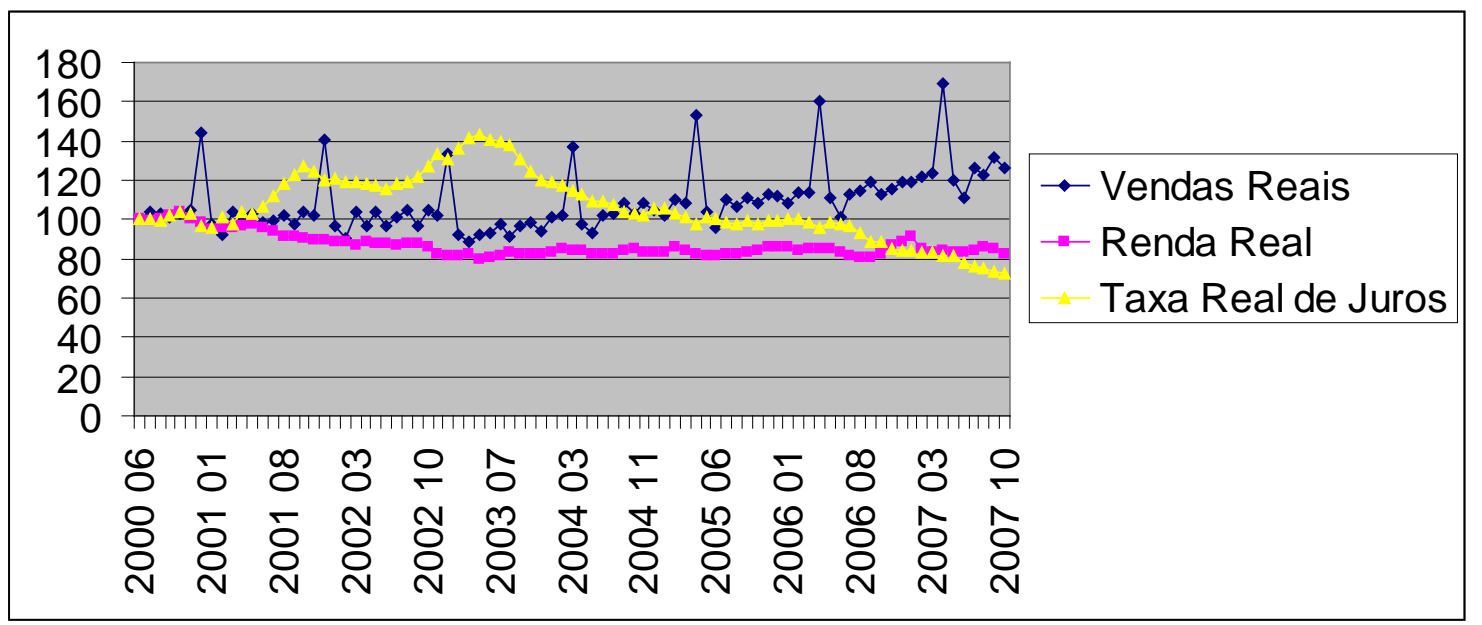

Grafico 1 - Evolução das Vendas Reais, Renda Real e Juros Reais (Junho de 2000 - Outubro de 2007)

Fonte: IPEA (2008)

Como o objetivo do trabalho é o de discutir a racionalidade das decisões do consumo e não propriamente examinar a evolução do consumo no período pós - Real, preferiu-se trabalhar com uma base de dados única, enfatizando as questões próprias ao processo decisório. Por essa razão que, a despeito de se comentar amplamente a evolução do consumo nos anos subseqüentes à edição do Plano, centrou-se a análise em um horizonte mais restrito.

Na próxima seção são apresentados os aspectos teóricos que sustentam o debate sobre o processo decisório dos indivíduos em suas atividades de consumo. Duas visões são contrapostas por meio da descrição dos trabalhos e dos achados de autores que defendem ou se opõem à idéia da racionalidade.

\subsection{Racionalidade e as Decisões de Consumo}

A idéia da racionalidade é uma questão abrangente. O estudo da história econômica revela que o conceito de racionalidade sob a perspectiva sócio-economica associada à idéia de comportamento razoável, isto é, em um contexto mais amplo, deu lugar a um entendimento bem mais específico, ou seja, a de racionalidade como a orientação seguida pelos indivíduos para a maximização da satisfação, expressa precisamente na forma de utilidade (ZAFIROVSKI, 2008).

A teoria econômica clássica supõe racionalidade dos agentes econômicos. A racionalidade do ponto de vista do consumidor implica na idéia de que os indivíduos baseiam suas escolhas com base nos preços relativos dos bens e serviços colocados à disposição, na renda e nas preferências representadas pelas denominadas funções utilidade.

Em uma visão simples os indivíduos contrapõem seus desejos, mapa de utilidade, com as possibilidades impostas pelas restrições de renda, conhecidos os vetores de preços.

A partir dessa concepção, a teoria econômica construiu formas mais complexas e adequadas de interpretação do processo de escolha dos indivíduos. Duas delas devem ser destacadas: a teoria da renda permanente e a hipótese de expectativas racionais.

As decisões econômicas em geral e, portanto, as de consumo são tomadas tendo em conta não apenas a experiência passada, mas também e, principalmente, o curso futuro suposto dos fatos, ou seja, as expectativas.

As expectativas há muito foram consideradas como elemento importante para explicar a dinâmica econômica. O próprio Keynes (1936) reconheceu essa importância, entretanto, 


\section{RACIONALIDADE E IRRACIONALIDADE NO PROCESSO DECISÓRIO DE CONSUMO}

tratava as expectativas de modo absolutamente exógeno. Não se dispunha de um modelo que incorporasse a formação das expectativas endogenamente.

No que diz respeito à função consumo além da contribuição de Keynes (1936), um dos mais importantes estudos sobre o assunto é o trabalho de Ando e Modigliani (1969). Nesse trabalho, os autores formulam a hipótese sobre o ciclo de vida dos indivíduos e seus reflexos sobre o consumo. Supõe-se que os indivíduos dispõem de um fluxo de renda com o seguinte perfil: relativamente baixo no começo da vida tendendo a crescer até atingir um valor máximo no meio da existência do indivíduo. Após manter-se por algum tempo nesta posição a renda passaria a cair até o final de sua vida.

Desse modo, no início da vida os indivíduos consumiriam mais do que a renda corrente permitiria. O consumo nesta etapa seria financiado pelo endividamento. Esse endividamento seria graduamente quitado pela fase posterior na qual a renda supera o consumo corrente. Nesse período os recursos não apenas cobririam as dívidas, mas também financiariam o consumo corrente e o consumo futuro mantido graças à poupança obtida nesta etapa.

Um importante avanço teórico nesta direção especificamente contemplando as decisões de consumo foi dado com o trabalho pioneiro de Milton Friedman sobre a função consumo (FRIEDMAN, 1957).

Friedman (1957, p. 21) levantou a hipótese da renda permanente. Para ele a renda em determinado período de tempo poderia ser desdobrada em dois componentes: a renda permanente $\left(y_{\mathrm{p}}\right)$ e a renda transitória $\left(y_{\mathrm{t}}\right)$.

A renda permanente corresponderia às expectativas de ganhos considerando os atributos individuais, tais como, a formação, as habilidades pessoais, o segmento de atuação, etc. Em outras palavras, a renda permanente diz respeito aos valores que os indivíduos estimam dispor em função de um conjunto específico de características pessoais.

O segundo componente formador da renda corrente é a renda transitória condicionada pelos movimentos cíclicos da economia, ou seja, as melhores ou piores condições momentâneas.

Associa-se aos dois componentes formadores da renda, o consumo, igualmente dividido em duas partes: o consumo permanente $\left(C_{P}\right)$ e o consumo transitório $\left(C_{T}\right)$. $O$ consumo transitório está ligado a situações particulares, tais como, por exemplo, o surgimento de uma oportunidade excelente ou a necessidade de suspensão das compras planejadas por conta de uma doença.

Sinteticamente pode-se dizer que de acordo com a hipótese da renda permanente, os indivíduos tendo em conta sua expectativa de vida maximizam a utilidade tomando como elemento restritor não apenas a renda, mas também a riqueza acumulada.

O consumo por sua vez pauta-se pela renda permanente. Segundo essa concepção, como salienta Hall (1978), o consumo seguiria um processo autoregressivo, eventualmente na forma de um movimento random walk, a partir do qual os valores futuros seriam definidos exclusivamente com base no consumo corrente.

De outro modo, como o indivíduo é racional e procura maximizar a utilidade, o consumo corrente por si só incorporaria todo o conjunto informacional definido pela renda permanente. Desse modo, assumindo para simplifcar apenas dois períodos, a condição de maximização seria dada pela seguinte condição:

$$
\text { (1) } u^{\prime}\left(C_{t}\right)=(R / \delta) E\left[u^{\prime}\left(C_{t+1}\right)\right]
$$

ou ainda: 


\section{(2) $\mathbf{u}^{\prime}\left(\mathrm{C}_{\mathrm{t}}\right) / \mathbf{R}=\mathbf{E}\left[\mathbf{u}^{\prime}\left(\mathrm{C}_{\mathrm{t}+1}\right)\right] / \delta$}

onde:

u' = utilidade marginal

$\mathrm{C}_{\mathrm{t}}=$ Consumo no período " $\mathrm{t}$ "

$\mathrm{C}_{\mathrm{t}+1}=$ Consumo no período " $\mathrm{t}+1$ ”

$\mathrm{R}=$ Taxa de juros

$\delta=$ Taxa de desconto

$\mathrm{E}=$ Esperança do consumo no período $\mathrm{t}+1$ dado um determinado conjunto de informações

O segundo membro da expressão (2) mostra que o valor da esperança marginal do consumo no período " $\mathrm{t}+1$ " descontado pela taßa deve ser igual à utilidade marginal do consumo corrente pela taxa de juros. Admitindo-se que $\mathrm{R}=\delta$, pode-se reescrever:

$$
\text { (3) } u^{\prime}\left(C_{t}\right)=E\left[u^{\prime}\left(C_{t+1}\right)\right]
$$

Desse modo, se a igualdade não se mantiver, o consumidor pode simplesmente aumentar sua satisfação transferindo ou antecipando consumo entre os dois períodos.

Supondo que as variações de utilidade sejam pequenas de um período para o outro pode - se escrever a trajetória do consumo entre “ $t$ - 1 ” e “ $t$ ” do seguinte modo:

$$
\text { (4) } C_{t}=\mu \cdot\left(C_{t-1}\right)+\xi_{t}
$$

onde:

$\mu$ = parâmetro dependente da taxa de juros e da taxa de desconto;

$\xi_{\mathrm{t}}=$ termo aleatório.

A expressão (4) afirma que o consumo corrente é uma função do consumo no período anterior mais um termo aleatório. Conhecido, portanto, o consumo do período anterior e as taxas de juros e de desconto, o consumo no período seguinte seria determinado implicitamente. Em outras palavras, o consumo corrente seria ajustado ou adaptado com base no consumo observado no período anterior pela interveniência do parâmetro $\mu$. De modo mais simples supondo comportamento quadrático para a função utilidade, a expressão (4) seria assim modificada:

$$
\text { (5) } \mathrm{C}_{\mathrm{t}}=\mathrm{C}_{\mathrm{t}-1}+\xi_{\mathrm{t}}
$$

O consumo seria garantido pela renda permanente. Estudando o comportamento da poupança agregada frente a variações da renda, Flóden (2008) conclui que as oscilações da poupança têm o propósito fundamental de suavizar o consumo, ou seja, em consonância com a hipótese da renda permanente.

A hipótese das expectativas racionais, por sua vez, foi amplamente discutida a partir dos anos 70. A estagnação econômica acompanhada de altos índices de inflação apresentou-se como um novo fenômeno. A conjugação de estagnação com inflação veio a ser conhecida como estagflação (DOELLINGER, 1975).

Os economistas keynesianos consideravam que uma política monetária mais ativa produziria movimentos importantes em termos de produto e emprego, pois os agentes 


\section{RACIONALIDADE E IRRACIONALIDADE NO PROCESSO DECISÓRIO DE CONSUMO}

econômicos incorporariam as informações suscitadas a partir do novo cenário de modo muito lento. Lucas e Sargent (1978), apoiados nas idéias de Friedman (1957), assinalaram que os indivíduos agem racionalmente e, portanto, consideravam que uma expansão monetária seria rapidamente antecipada não afetando a taxa natural de desemprego.

A hipótese das expectativas racionais é sinteticamente bastante simples. Os agentes econômicos, consumidores e produtores não desperdiçam informações e utilizam os elementos gerados pela própria dinâmica econômica para aprimorar o processo decisório. Isto não significa que os erros deixam de existir, porém segundo a hipótese das expectativas racionais os indivíduos não cometem sistematicamente os mesmos erros.

Há, entretanto, uma importante diferença entre o mecanismo adaptativo das expectativas e a idéia das expectativas racionais. No primeiro os agentes realizam um conjunto de ajustes de acordo com a dinâmica da própria aprendizagem. Na hipótese das expectativas racionais supõe-se que os indivíduos têm um conjunto subjacente de valores e informações que os permite detectar e antecipar mudanças do cenário econômico. Lucas (1986) entende a ciência econômica como o conjunto estável de regras de decisão formado a partir de um processo adaptativo. Portanto, trata-se de um processo de aprendizagem amplo capaz de suscitar regras comportamentais genéricas que induzem a movimentos antecipatórios de ajuste.

Pode-se talvez ilustrar o funcionamento do mecanismo adaptativo e a hipótese propulsora das expectativas racionais por meio do trabalho de Wunderick (1995). Neste trabalho estuda-se a decisão dos casais em relação à formação da família, ou seja, o número de filhos e o tempo mais adequado para tê-los. O trabalho conclui que o número de filhos e o tempo mais apropriado dependem de uma avaliação do futuro do casal, da experiência acumulada, particularmente com o primeiro filho, e também das preferências intertemporais das pessoas.

Desse artigo pode-se inferir que a avaliação do futuro e as preferências intertemporais poderiam ser sustentadas pelas condições econômicas e sociais gerais. Essas condições de contorno se distinguem em função do grau de desenvolvimento das diferentes sociedades e implicam em preferências difereciadas relativamente ao número de filhos e à data mais propícia para ampliar a família. Esses aspectos definem, portanto, as dimensões subjacentes nessa questão e, poderiam ser definidas como os elementos condicionadores na racionalização das expectativas. Porém, tanto o número de filhos como o correspondente período do nascimento se revelaram influenciados pela experiência dos casais com o primeiro filho. Tal experiência atuaria como mecanismo adaptador na formação da família.

As expectativas são, na verdade, um aspecto presente em toda a teoria econômica. Entretanto, até os trabalhos de Lucas (1986), essa questão não havia sido formulada de modo absolutamente claro.

Lucas (1986) teve o mérito de incorporar na modelagem econômica a formação das expectativas por parte dos agentes econômicos. Na verdade, a simplicidade revolucionária da hipótese das expectativas racionais tem motivado alguns autores a investigar a gênese deste importante princípio.

Embora o trabalho de Lucas seja considerado seminal, a literatura usualmente aponta a contribuição de Muth (1961) como o primeiro estudo a valer-se da idéia da racionalidade na formação das expectativas.

Porém, provavelmente corroborando a idéia de que o princípio das expectativas racionais é bastante natural, Keuzenkamp (1991) registra que a primeira visão sobre esse assunto foi, na verdade, traduzida por Tinbergen quase 30 anos mais cedo que o trabalho de Muth, ou seja, 1932. 
Keuzenkamp (1991) mostra que o trabalho de Tinbergen (1932) estruturava-se considerando que consumidores e produtores maximizavam suas respectivas funções de utilidade e de lucro, considerando um determinado horizonte de tempo e tendo em conta os preços esperados. O resultado dessa ação independente, pressupunha o modelo, levaria a resultados inconsistentes, obrigando a realização de ajustes de modo que os excessos de oferta e demanda se eliminariam, compatibilizando os diferentes planos no período corrente.

Comparando as contribuições de Tinbergen (1932) e Muth (1961), Keuzenkamp identifica grande semelhança e, considera que a idéia das expectativas racionais poderia ter sido conhecida bem antes, não tivesse o trabalho de Tinbergen sido redigido em alemão (KEUZENKAMP, 1991)

De qualquer modo, Muth expressou explicitamente a idéia de que os agentes agem formando expectativas com base no conjunto de informações disponíveis. Entretanto, a exemplo do trabalho de Tinbergen, as idéias de Muth, pelas razões apresentadas permaneceram inexploradas até o advento das contribuições de Lucas a partir de 1962. Em 1969, Lucas e Rapping com base no mecanismo de formação das expectativas, estudam a dinâmica do mercado de trabalho e sustentam a tese, defendida por Friedman, de uma taxa natural de desemprego. A modelagem proposta pelos dois autores torna-se a base sobre a qual se sustentaria o posterior desenvolvimento da economia neoclássica moderna (LUCAS; RAPPING, 1969).

Como se viu, embora a hipótese das expectativas racionais apóie-se parcialmente na idéia das expectativas adaptadas, há importantes diferenças entre as duas concepções. As expectativas adaptadas fundamentam-se na idéia de um processo contínuo de ajustamento orientado pela aprendizagem. Nas idéias suscitadas pela hipótese das expectativas racionais os agentes são capazes de identificar relações estocásticas estáveis entre as variáveis de interesse.

Glazer et al. (1990) comparam o processo decisório de indivíduos submetidos a um processo de gestão simulada. Utilizando um jogo de empresas, os autores comparam as decisões tomadas examinando se o processo de escolha das alternativas dos jogadores fundamenta-se na idéia de expectativas adaptadas ou na hipótese de expectativas racionais.

Aplicando o jogo Markstrat para alunos do MBA da Universidade de Columbia, os autores sustentam que não se evidenciaram elementos que corroborassem a idéia das expectativas adaptadas. A simulação revelou, entretanto, uma baixa variância nas estimativas das variáveis relevantes, indicando apoio à hipótese das expectativas racionais. Entretanto, a suposição que a atuação conjunta dos indivíduos eliminaria possíveis vieses não se confirmou. Portanto, no caso das expectativas racionais, às evidências apontadas pelos autores se sustentam apenas parcialmente (GLAZER et al., 1990).

Em oposição às idéias do comportamento racional dos indivíduos se coloca o pensamento de Simon (1957). Questionando os axiomas da racionalidade, Simon estabelece o conceito de racionalidade limitada. Para o referido autor os agentes econômicos não conseguem frequentemente tomar deciões ótimas, mas sim, simplesmente satisfatórias (princípio da racionalidade limitada). O principío se assenta na concepção de que os indivíduos fndamentam suas decisões em um conjunto limitado de informações.

Isso ocorre porque é impossível para um único indivíduo apresentar isoladamente um comportamento com alto grau de racionalidade. Neste contexto, Simon (1957) introduz o comportamento racional como sendo individualizado e uma função de propriedades psicológicas incluindo percepção, pensamento e aprendizagem.

A idéia da racionalidade limitada assume que para lidar com as complexidades do mundo real, um indivíduo constrói um modelo simplificado para cada situação. Isto está 


\section{RACIONALIDADE E IRRACIONALIDADE NO PROCESSO DECISÓRIO DE CONSUMO}

claramente em contradição com a teoria normativa que prescreve abordagens que buscam uma solução específica ideal ou ótima para um problema.

Nesta direção outros pesquisadores, notadamente Kahneman e Tversky (1979) abriram outra vertente de investigação. Esses autores salientaram que a necessidade de processar informações leva necessariamente a importantes e significativas simplificações na leitura do ambiente por parte dos decisores. Apoiando-se em experimentos os autores sustentam que o comportamento dos indivíduos se desvia frequentemente do que se poderia pontuar como racional.

Avançando na linha de Simon, Kahneman e Tversky (1979) consideram que os indivíduos se valem de estratégias fundamentadas em simplificações ou preconceitos que não conduzem necessarimente a uma situação de maximização da satisfação dos indivíduos. Tal concepção veio a ser conhecida na literatura como Teoria dos Prospectos.

A Teoria dos Prospectos qualifica um conjunto de iniciativas utilizadas pelas pessoas, como atalhos mentais, empregados inconcientemente pelos agentes nos mais diversos tipos de decisões (KAHNEMAN; TVERSKY, 1979). Por exemplo, o fenômeno da ancoragem em que os indivíduos norteiam suas decisões a partir de alguns poucos elementos, impedindo a compreensão mais ampla do contexto que conduziria a uma situação ótima. Outra dimensão é o denominado efeito framing, em que a maneira em que o problema é proposto interfere na escolha do decisor.

Para evidenciar esses processos mentais, Kahneman e Tversky (1972; 1979), bem como outros pesquisadores que vêm trilhando esse caminho, têm desenvolvido uma série de heurísticas, com o propósito de revelar a presença destes vieses, que conduzem a decisões apenas parcialmente racionais, ou mesmo irracionais

Os preços, por exemplo, segundo a teoria dos Prospectos representam na verdade valores de referência captados e processados pelos indivíduos dependendo de situações particulares. Por exemplo, os preços passados pagos geram referências que inflenciam o processo de valoração que se estabelece na seqüência. Nestas condições, um consumidor, pressupõe a mencionada teoria, estaria disposto a pagar um preço diferente pelo mesmo bem dependendo simplesmente dos valores pagos anteriormente. Ou ainda, o valor pago poderia ser considerado mais ou menos justo dependendo do contexto onde a transação ocorre. Se o bem fosse comprado, por exemplo, em uma loja que suscitasse maior sofisticação, considera a Teoria dos Prospectos, a disposição de dispêndio por parte do consumidor seria maior do que se o mesmo bem fosse adquirido em um local mais simples (SMITH; NAGLE, 1995).

Corroborando esta visão no tratamento de questões de financiamento, Gourville (1998) e Christensen (1986) salientam que as escolhas dos consumidores pelas opções entre diferentes planos são muito influenciadas pelo valor das parcelas.

Resultado semelhante foi encontrado por Queiroz (2007). Trabalhando com dados primários coletados a partir de questionários aplicados a consumidores, identificou-se, no referido estudo, correlação significativa entre o valor da parcela e a preferência dos consumidores pelas alternativas de crédito nos diferentes grupos sócio-econômicos estudados no município de São Paulo. De outro modo o resultado também deste trabalho corrobora a tese da importância da forma de apresentação do parcelamento no processo decisório de compra dos indivíduos.

A seguir propõe-se a abordagem analítica que se utilizará com o objetivo de avaliar as idéias anteriormente expressas no âmbito da economia brasileira em um subperíodo posterior a edição do Plano Real.

\section{MODELO ANALÍTICO E BASE DE DADOS}


O consumo mensal dos indivíduos é ao longo de um determinado período de tempo, como revelou a Figura 1 anteriormente mostrada, influenciado obviamente por movimentos sazonais do mercado. Esses movimentos são explicados pelas próprias estações do ano e a ocorrência de datas especiais como festas do final do ano, dia das mães, dia dos namorados, etc.

Trabalhando com dados sujeitos a movimentos sazonais bem definidos, como é o caso das vendas de varejo, Enders (1995, p. 113) propõe três modelos analíticos com o objetivo de descrever a evolução do fluxo de turistas com destino à Espanha: autoregressivo, médias móveis - aditivo e médias móveis multiplicativo. Os modelos sugeridos por Enders são apresentados com a utilização dos operadores de defasagem "L". Por exemplo, o operador $\mathrm{L}^{\mathrm{k}}$ aplicado à variável $\mathrm{y}_{\mathrm{t}}, \mathrm{L}^{\mathrm{k}}\left(\mathrm{y}_{\mathrm{t}}\right)$, indica que a variável “ $\mathrm{y}$ ” é defasada em " $\mathrm{k}$ ” períodos, isto é $\mathrm{y}_{\mathrm{t}}$ k.

Com o propósito de testar a hipótese da renda permanente, ou seja, da racionalidade, modelou-se o consumo considerando três especificações alternativas, nas três inclui-se as variáveis taxa de juros $(\mathrm{TJ})$ e renda $(\mathrm{R})$.

Espera-se rejeitar a significância dos parâmetros correspondentes tanto a taxa de juros como a renda, isto porque caso o consumidor se comporte racionalmente, os impactos tanto de uma variável como de outra já se encontrariam devidamente incorporados e refletidos no consumo corrente. De outro modo, os valores do consumo dos períodos passados, condicionados pelas variáveis renda e taxa de juros, deveriam explicar, verificando-se a hipótese de racionalidade, os movimentos do consumo corrente.

Os três modelos abaixo não expressam as relações entre as variáveis em nível. Tomouse a primeira diferença da variação das vendas, renda e juros em doze meses. As variáveis em nível não são estacionárias, o que implicaria em regressões espúrias (ENDERS, 1995, p.216). Da forma como se especificou obteve-se a estacionariedade das referidas variáveis conforme apurado pela aplicação do teste Dickey Fuller.

(i) $\left(1-L^{12}\right) \cdot(1-L) \cdot\left(1-a_{12} \cdot L^{12}\right) \cdot y_{t}=\beta_{r} \cdot\left(1-L^{12}\right) \cdot(1-L) \cdot R+\beta_{j} \cdot\left(1-L^{12}\right) \cdot(1-L) \cdot T J+\left(1+\beta_{1}\right) \cdot \varepsilon_{t}$

(ii) $\left(1-L^{12}\right) \cdot(1-L) \cdot y_{t}=\beta_{r} \cdot\left(1-L^{12}\right) \cdot(1-L) \cdot R+\beta_{j} \cdot\left(1-L^{12}\right) \cdot(1-L) T J+\left(1+\beta_{1} \cdot L\right)\left(1+\beta_{12} \cdot L^{12}\right) \cdot \varepsilon_{t}$

$$
\text { (iii) }\left(1-L^{12}\right) \cdot(1-L) \cdot y_{t}=\beta_{r} \cdot\left(1-L^{12}\right) \cdot(1-L) \cdot R+\beta_{j} \cdot\left(1-L^{12}\right) \cdot(1-L) \cdot T J+\left(1+\beta_{1} \cdot L+\beta_{12} L^{12}\right) \cdot \varepsilon_{t}
$$

Como já se assinalou os dados utilizados na estimação dos modelos i, ii e iii foram obtidos todos da mesma fonte, ou seja, o IPEA - Instituto de Pesquisa Econômica Aplicada e referem-se ao período de junho de 2000 a outubro de 2007 (IPEADATA, 2008).

\section{RESULTADOS}

Os três modelos foram estimados pelo método dos mínimos quadrados, valendo-se do software EViews. Os resultados referentes aos correspondentes parâmetros encontram-se apresentados na Tabela 1. A segunda coluna mostra os mencionados parâmetros. Abaixo de cada um dos valores foi explicitada a significância das estimativas. Colocou-se ainda para cada modelo, o indicador Akaike (AIC) e Schwarz (SBC). Finalmente encontra-se devidamente explicitada a soma dos quadrados dos resíduos para cada um dos três modelos (SQR). Nos três modelos os resíduos se revelaram estacionários de acordo com o teste Dickey - Fuller. 
RACIONALIDADE E IRRACIONALIDADE NO PROCESSO DECISÓRIO DE CONSUMO

\begin{tabular}{|c|c|c|c|}
\hline Parâmetro & Modelo “i” & Modelo “ii” & Modelo “iii” \\
\hline $\boldsymbol{\beta}_{\mathbf{j}}$ & $\mathbf{- 0 . 2 6 6 0 5 0}$ & $\mathbf{- 0 . 2 3 3 5 1 1}$ & $\mathbf{- 0 . 2 1 7 3 9 9}$ \\
& $(0.0000)$ & $(0.000)$ & 0.0014 \\
\hline $\boldsymbol{\beta}_{\mathrm{r}}$ & $\mathbf{0 . 1 1 0 2 3 0}$ & $\mathbf{0 . 1 0 4 1 7 8}$ & $\mathbf{0 . 1 3 9 3 5 0}$ \\
& $(0.3365)$ & $(0.3558)$ & 0.1991 \\
\hline $\mathbf{A}_{12}$ & $\mathbf{- 0 . 2 5 4 2 8 8}$ & - & - \\
& $(0.0342)$ & & $\mathbf{- 0 . 4 3 8 8 6 1}$ \\
\hline $\boldsymbol{\beta}_{12}$ & - & $\mathbf{- 0 . 8 8 4 5 1 7}$ & 0.0000 \\
\hline $\boldsymbol{\beta}_{1}$ & $\mathbf{- 0 . 6 7 5 3 3 4}$ & $\mathbf{- 0 . 6 5 0 8 7 5}$ & $\mathbf{- 0 . 5 3 5 8 3 2}$ \\
& $(0.0000)$ & $(0.0000)$ & 0.0000 \\
\hline AIC & -4.670615 & -5.003634 & -4.724599 \\
\hline SBC & $-\mathbf{- 4 . 5 3 5 6 8 5}$ & $\mathbf{- 4 . 8 8 0 9 6 4}$ & $-\mathbf{4 . 6 0 1 9 2 9}$ \\
\hline SQR & $\mathbf{0 . 0 3 0 9 7 4}$ & $\mathbf{0 . 0 2 6 8 8 9}$ & $\mathbf{0 . 0 3 5 5 4 3}$ \\
\hline
\end{tabular}

Quadro 1 - Resultados dos Modelos Estimados

O primeiro aspecto a ser notado é que independentemente do modelo escolhido o coeficiente correspondente a taxa de juros é sempre altamente significativo. Por outro lado, nos mesmos três modelos, as variações da renda não se mostraram relevantes na explicação das flutuações do consumo. Esse último resultado de certa forma era esperado tendo em vista as baixas variações da renda constatadas no período no qual as funções foram determinadas. Como se viu na Figura 1, da seção dois, depois de uma queda do início do período até o final de 2002, a renda real média do pessoal ocupado manteve-se praticamente inalterada até do final do período de observação, ou seja, outubro de 2007.

As variações da taxa de juros, por outro lado, se mostraram importantes na explicação das flutuações do consumo. Os erros passados expressos nos parâmetros $\boldsymbol{\beta}_{\mathbf{1}}$ e $\boldsymbol{\beta}_{\mathbf{1 2}}$, bem como os valores defasados representados pelo coeficiente $\mathbf{a}_{12}$, do modelo i, não são suficientes para explicar as alterações do consumo, caso fossem o parâmetro $\boldsymbol{\beta}_{\mathbf{j}}$, associado a variável taxa de juros, não deveria se revelar significativo como indicado nos resultados apresentados nos três modelos.

A taxa de juros não apenas é significativa como tem um peso relativo elevado. Como todas as variáveis estão mensuradas em índices fixados na mesma base o tamanho dos parâmetros, desde que significativo, fornece um indicador da importância das variáveis explicativas. Como se vê, as alterações da taxa de juros têm uma importância substancial nas variações do consumo.

Em síntese pode-se dizer que a hipótese de racionalidade nas decisões de consumo traduzida como a capacidade dos indivíduos de ajustarem seu comportamento em função exclusivamente da experiência passada, não se mostrou sustentável com base nas evidências levantadas. Ou seja, embora a renda não tenha se revelado significativa, o que condiz com a hipótese de racionalidade, as flutuações da taxa de juros demonstram inequivocamente que a capacidade de realização de ajustes por parte dos indivíduos no padrão de consumo com base exclusivamente nos valores passados não encontra apoio nos dados levantados. Deve-se ainda salientar mais uma vez que a não significância da renda pode ser devida ao período de tempo considerado, no qual a renda tevê pouca flutuação.

\section{CONSIDERAÇÕES FINAIS}


A discussão do comportamento racional dos indivíduos é uma questão frequentemente discutida na literatura econômica. Duas visões a respeito desta questão são relativamente nítidas no desenvolvimento teórico.

A primeira baseada nos argumentos da teoria clássica sustenta que os indivíduos em suas decisões maximizam os benefícios líquidos. Em princípio disposições (utilidade) e disponibilidade (restrições) são aspectos compatibilizados de modo a propiciar ao decisor o melhor resultado possível. Nesta perspectiva, embora os agentes econômicos cometam erros em suas previsões, supõe-se que a experiência passada permite que os indivíduos incorporem ao conjunto de informações esses desvios, reduzindo consequentemente as variações nos períodos seguintes dos valores observados em relação a um determinado comportamento médio.

A segunda visão associada à discussão da racionalidade dos indivíduos apóia-se nas idéias seminais de Simon (1957) e o princípio da racionalidade limitada. Para ele os indivíduos não conseguem por uma série de limitações ambientais e psicológicas tomar decisões realmente ótimas. Nestas condições os indíduos são forçados a decidir com base em um conjunto limitado de elementos, razão esta pela qual contentam-se apenas com resultados satisfatórios. Desdobramentos desta perspectiva analítica se materializam nos trabalhos de Kanneman e Tversky (1972; 1979).

Esses conceitos aplicam-se obviamente às decisões de consumo. De um lado, posicionam os que defendem que os indivíduos ajustam seu comportamento em função dos valores passados do próprio consumo, bem como dos eventuais erros cometidos. De outro modo, admitindo-se a racionalidade das decisões dos indivíduos os registros passados do consumo explicariam totalmente as variações do consumo presente.

O propósito fundamental do presente trabalho foi o de examinar essas duas propostas conceituais de acordo com a experiência recente da economia brasileira. Os dados sobre os quais se apoiaram três modelos parecem indicar que a hipótese da racionalidade limitada é a que melhor explica o comportamento do consumo no Brasil.

Uma limitação do trabalho é, sem dúvida, o período que certamente deveria ser mais longo. Obviamente esta colocação enseja uma extensão do trabalho examinando o comportamento das mesmas variáveis em horizonte mais amplo. De qualquer modo, trabalhando neste período mais dilatado, e consequentemente dando a oportunidade para que a renda flutue mais, é possível e, talvez bem provável, que não apenas a taxa de juros se revele significativa, mas também a própria renda. Isto reforçaria ainda mais as conclusões do presente trabalho. A base desta ilação é que, embora os coeficientes da renda não tenham se mostrado significativos, nos três modelos os parâmetros apresentam-se com sinal positivo como era de se esperar de acordo com a hipótese da limitação da racionalidade no processo decisório dos agentes econômicos.

\section{REFERÊNCIAS}

ANDO, A.; MODIGLIANI, F. Econometric Analysis of Stabilization Policies. The American Economic Review, v.59, n. 2, p. 296-314, 1969.

BAUMOL, W.J. Entrepreneurship in economic theory. The American Economic Review, v. 58, p. $64-71,1968$.

BROCHAUS, R.H. Sr. The psychology of the entrepreneur. In: KENT, C.A.; SEXTON, D.L. \& VESPER, K.H. (Eds.) Encyclopedia of entrepreneurdhip. Englewood Cliffs, N.J., Prentice-Hall, p. 39 - 57, 1982. 
BYGRAVE, W.D. The entrepreneurship paradigm (I): a philosophical look at its research methodologies. Entrepreneurship: Theory \& Practice, p. 7- 26, Fall 1989a.

.The entrepreneurship paradigm (II): chaos and catastrophes among quantum jumps? Entrepreneurship: Theory \& Practice, p. 7- 30, Winter 1989b.

. Theory building in the entrepreneurship paradigm. Journal of Business Venturing, v. 8, n. 3, p. 255 - 280, 1993.

CHRISTENSEN, C. Decision Theory and consumer behavior. Ohio: Ohio University, 1986.

DOELlinger, C. von. Agumas notas sobre a crise econômica internacional. Pesquisa e Planejamento Econômico, v.(5), n. 1, p.231 - 260, IPEA - Rio de Janeiro, Junho 1975.

ENDERS, W. Applied econometric time series. New York: John Wiley \& Sons, Inc., 1995.

FLODÉN, M. Aggregate savings when individual income varies. Review of Economic Dynamics, v.11, n.1, p. 70-82, 2008.

FRIEDMAN, M. A Theory of the Consumption Function. Princeton University Press, Princeton, 1957.

GLAZER, R.; STECKEL, J.H.; WINER, R.S. Judgmental forecasts in a competitive environment: Rational vs. adaptive expectations. International Journal of Forecasting, v.6, n. 2, p. 149-162, 1990.

GIAMBIASI, F. (2005). Estabilização, reformas e desequilíbrios macroeconômicos: os anos FHC (1995-2002). In: Giambiasi, F. et al. (Org.). Economia brasileira contemporânea (1945-2004). (pp.166-195). Rio de Janeiro: Editora Campus.

GOURVILLE, J. T. Pennies-a-day: The effect of temporal reframing on transaction evaluation. Journal of Consumer Research, v.24, n. 4, p.395-410, 1998.

HALL, R.E. Stochastic implications of the life cycle-permanent income hypothesis: theory and evidence. Journal of Political Economy, v.86, n.6, p. 971-987, 1978.

IPEA - Instituto de Política Econômica Aplicada. Consumo e Vendas. Disponível em: www.ipeadata.gov.br/ipeaweb.dll/ipeadata?SessionID=590021753\&Tick=1216065556265\& VAR_FUNCAO=RedirecionaFrameConteudo\%28\%22iframe_dados_m.htm\%22\%29\&Mod= M>. Acesso em: 23/05/2008.

KAHNEMAN, D.; TVERSKY, A. Subjective probability: a judgment of representativeness. Cognitive Psychology, v.3, pp. 430-454, 1972.

291, 1979.

. Prospect Theory: an analysis of decision under risk. Econometrica, v.47, p.263- 
KEUZENKAMP, H.A. A Precursor to Muth: Tinbergen's 1932 Model of Rational Expectations. The Economic Journal, v.101, n. 408, p. 1245-1253, 1991.

KEYNES, John Maynard. A Teoria Geral do Emprego, do Juro e da Moeda. Editora Atlas, 1990. Original, 1936.

LUCAS, R.E., Jr. Adaptative behavior and economic theory. The Journal of Business, v.59, n.4, p. s401 - s426, 1986.

LUCAS, R.E., Jr.; RAPPING, L.A. Real Wages, Employment, and Inflation. The Journal of Political Economy, v.77, n. 5, p. 721-754, 1969.

LUCAS, R.E., Jr.; SARGENT, T.J. New' Explanations of the Persistence of Inflation and Unemployment. In: After the Phillips Curve: Persistence of High Inflation and High Unemployment. Boston, Mass.: Federal Reserve Bank of Boston, 1978.

MUTH, J.F. Rational Expectations and the Theory of Price Movements. Econometrica, v. 29, n. 3, p.315-335, 1961.

QUEIROZ, R.S.B. Processo de Tomada de Decisão na Aquisição de Crédito e Preferências entre Alternativas de Financiamento no Varejo. São Paulo, 2007. 105f. Dissertação (Mestrado em Administração) - Programa de Pós-Graduação em Economia, Departamento de Economia, Faculdade de Economia, Administração e Contabilidade da Universidade de São Paulo, 2007.

SCHUMPETER, J.A. The Theory of Economic Development. Tradução de: Theorie der wirtschftlichen Entwicklung. Reprint. Originally published: Cambridge, Mass.: Harvard University Press, 1934. (Harvard economics studies v.46) 1983. Ed. 10, 2004.

SMITH, G.E.; NAGLE, T.T. Frames of Reference and Buyer`s Perception of Price and Value. California Management Review, v. 38, n. 1, p. 98-116, 1995.

SIMON, H.A. Models of man. New York: John Wiley and Sons, 1957.

TIMBERGEN, J. do original "Ein Problem der Dynamic" Zeitschriftfiur Nationalekonomie, III. Bd., 2.H.,p. 169-84, 1932. In: KEUZENKAMP, H.A. A Precursor to Muth: Tinbergen's 1932 Model of Rational Expectations. The Economic Journal, v.101, n. 408, p. 1245-1253, 1991.

TVERSKY, A.; KAHNEMAN, D. Belief in the law of small numbers. Psychological Bulletin, v.76, pp. 105-110, 1971. 207-232, 1973. Availability: a heuristic for judging frequency. Cognitive Psychology, v.5, pp. . Judgment under uncertainty: Heuristics and biases. Science, v.185, pp. 11241131, 1974. 
ZAFIROVSKI, M. Classical and neoclassical conceptions of rationality - findings of an exploratory survey. Journal of Socio-Economics, v.37, n.2, p. 789-820, 2008.

WUNDERINK, S.R. Is family planning an economic decision? Journal Economic Psychology, v.16, n. 3, p.377-392, 1995. 